\title{
Arecibo imaging of compact high-velocity clouds
}

\author{
W. B. Burton ${ }^{1}$, R. Braun ${ }^{2}$, and J. N. Chengalur ${ }^{3}$ \\ 1 Sterrewacht Leiden, PO Box 9513, 2300 RA Leiden, The Netherlands \\ 2 Netherlands Foundation for Research in Astronomy, PO Box 2, 7990 AA Dwingeloo, The Netherlands \\ 3 National Centre for Radio Astrophysics, Post Bag 3, Ganeshkind PO, Pune, Maharashtra 411 007, India
}

A\&A, 369, 616-642 (2001), DOI: 10.1051/0004-6361:20010162

\begin{abstract}
An arithmetic error in the numerical values of outer disk H I exponential scale-lengths in late-type dwarf galaxies is corrected. None of the other derived quantities in this publication are affected by this error. In particular, the distance estimates to the objects studied based on the mean outer disk scale-length remains in the range 320 to $730 \mathrm{kpc}$.
\end{abstract}

Key words. ISM: atoms - ISM: clouds - galaxy: evolution - galaxy: formation - galaxies: dwarf galaxies: local group

\section{Outer disk scale-lengths in late-type dwarf galaxies}

We have discovered an arithmetic error in our recent publication (Burton et al. 2001), corresponding to exactly a factor of ten in the value of the exponential scale lengths, $h_{\mathrm{e}}$, of outer disk H I in the sample of 15 nearby late-type dwarf galaxies which we took from Swaters (1999). The mean exponential scale-length value for this sample is actually $h_{\mathrm{e}}=1.17 \pm 0.44 \mathrm{kpc}$. The corresponding scalelength of the modified Bessel function of order $1, h_{\mathrm{B}}$, which we use to represent the $\mathrm{H}$ I edge profiles of the compact high velocity clouds we studied, is $10 \%$ smaller, namely $h_{\mathrm{B}}=1.06 \pm 0.40 \mathrm{kpc}$. These are the numerical values which should appear throughout the manuscript, specifically (1) in the Abstract, (2) in the caption and column heading of Table 2, (3) in the last paragraph of Sect. 8, (4) in the axis labeling and caption of Fig. 16 and (5) in the last paragraph of Sect. 9. None of the other derived quantities in the manuscript were affected by this error. In particular, the distance estimates to the objects studied based on the mean outer disk scale-length remains in the range 320 to $730 \mathrm{kpc}$.

\section{References}

Burton, W. B., Braun, R., \& Chengalur, J. N. 2001, A\&A, 369, 616

Swaters, R. A. 1999, Ph.D. Dissertation, University of Groningen

Send offprint requests to: R. Braun, e-mail: rbraun@nfra.nl 